Heike Wiese (Potsdam)

\title{
Das Potenzial multiethnischer Sprechergemeinschaften
}

\begin{abstract}
Der Beitrag behandelt den Sprachgebrauch in multiethnischen Sprechergemeinschaften im urbanen Raum. Ich zeige, dass die Varietät, die sich hier entwickelt, als neuer Dialekt des Deutschen verstanden werden kann. Dieser Dialekt ist gekennzeichnet durch Charakteristika auf lexikalischer und grammatischer Ebene, die auf systematische Muster sprachlicher Variation und sprachlichen Wandels hinweisen, und erhält durch seine Sprechergemeinschaft mit vielen (aber nicht nur) mehrsprachigen Sprecher/inne/n eine besondere sprachliche Dynamik. Ich diskutiere zwei Beispiele, intensivierend gebrauchtes ,voll" und monomorphematisches, existenzanzeigendes ,gib $(t) s$ ", die die quantitative Expansion bzw. die Weiterentwicklung und den qualitativen Ausbau von Phänomenen illustrieren, die auch aus anderen Varietäten des Deutschen bekannt sind. Der multiethnische urbane Dialekt, der hier entsteht, spiegelt damit Entwicklungstendenzen des Deutschen wieder, die in einigen Fällen zusätzlich durch Sprachkontaktphänomene gestützt werden können.
\end{abstract}

\section{Neue multiethnische Sprechergemeinschaften im urbanen Raum}

Die Gesellschaften im modernen Europa sind, insbesondere im urbanen Raum, heute durch ethnisch und sprachlich gemischte Populationen charakterisiert. In Folge von Immigration sind multiethnische Wohngebiete entstanden, in denen Menschen unterschiedlicher Herkunft und unterschiedlicher Erst- und Zweitsprachen zusammenleben. In Deutschland hatte 2009 knapp ein Fünftel $(19,2 \%)$ der Bevölkerung einen so genannten „Migrationshintergrund", 2010 lebten rund 31\% der minderjährigen, ledigen Kinder in Deutschland in einer Familie mit Migrationshintergrund, wobei in urbanen Gebieten der Anteil höher liegt. In Großstädten mit mehr als 500.000 Einwohnern wächst fast jedes zweite minderjährige Kind (46\%) in einer Familie mit Migrationshintergrund und damit in einer potenziell mehrsprachigen Familie auf. ${ }^{2}$ Es handelt sich hierbei also um kein Randphänomen, sondern um einen substanziellen Anteil der Bevölkerung, der die sprachliche Landschaft um vielfältige Ressourcen bereichert und dazu

\footnotetext{
Quelle: Statistisches Bundesamt; Menschen mit Migrationshintergrund sind dort definiert als Personen, die nach 1949 auf das heutige Gebiet der Bundesrepublik Deutschland zugezogen sind, sowie alle in Deutschland geborenen Ausländer/innen und alle in Deutschland Geborenen mit zumindest einem zugezogenen oder als Ausländer/in in Deutschland geborenen Elternteil.

Zahlen für 2010; Quelle: Statistisches Bundesamt.
} 
führt, „dass die bundesrepublikanische Gesellschaft mehrsprachig ist - einfach weil ein nicht unerheblicher Teil der Bevölkerung mehrsprachig ist" (Schroeder 2007, S. 7).

Durch das Zusammenleben von Sprecher/inne/n unterschiedlicher (einschließlich deutscher) Herkunft entstehen im urbanen Raum neue, multiethnische und multilinguale Sprechergemeinschaften mit eigener sprachlicher Dynamik. Der Sprachenvielfalt auf Ebene der Sprachsysteme steht auf Sprecherebene neben einsprachig deutschen Sprecher/inne/n ein hoher Anteil mehrsprachiger Sprecher/innen gegenüber, die mit mindestens einer weiteren Sprache neben dem Deutschen aufgewachsen sind. Dies führt zur Entstehung neuer multiethnischer urbaner Varietäten, ${ }^{3}$ „urban vernaculars" (Rampton ersch.demn.), insbesondere in der sprachlich besonders dynamischen Gruppe der jugendlichen Sprecher/innen, aber, wie ich unten an einem Beispiel zeigen werde (Abschnitt 3.2), mit weiterem Verbreitungspotenzial.

Für diesen neuen Sprachgebrauch sind unterschiedliche Bezeichnungen verbreitet. Ich verwende in Anlehnung an eine Bezeichnung, die jugendliche Informant/inn/en in Berlin geprägt haben, den Begriff „Kiezdeutsch“. Diese Bezeichnung beinhaltet keine ethnische Eingrenzung und kann damit erfassen, dass es sich um eine Varietät handelt, die für ein bestimmtes alltägliches Wohnumfeld (Berlinisch „Kiez") charakteristisch ist und dort unabhängig von unterschiedlichen Herkunftssprachen (Türkisch, Arabisch, Deutsch, Kurdisch, ...) gesprochen wird.

\section{Kiezdeutsch als multiethnischer Dialekt des Deutschen}

Wie ich an anderer Stelle ausführlich argumentiert habe (Wiese 2012), kann Kiezdeutsch als neuer, multiethnischer Dialekt des Deutschen angesehen werden. Mit dieser Einordnung beziehe ich mich auf einen weiten Dialektbegriff, wie er seit den 1980ern insbesondere in Soziolinguistik und Variationslinguistik entwickelt wurde. Diese weite Auffassung von „Dialekt“ subsumiert sprachliche Varietäten, die eine bestimmte Sprechergruppe innerhalb einer größeren Sprachgemeinschaft charakterisieren; vgl. etwa eine häufig zitierte Definition von , ,dialect" in Trudgill (1992, S. 23; vgl. auch Chambers/Trudgill 1998):

a variety of language which differs grammatically, phonologically and lexically from other varieties, and which is associated with a particular geographical area and/or with a particular social class or status group.

Ich nehme hier eine Perspektive ein, die primär auf das sprachliche System abzielt und soziolinguistische Fragen des Gebrauchs an dieser Stelle vernachlässigt, und gehe von Varietäten aus. Zur Diskussion von Multiethnolekten als „Stil "c vs. „Varietät" vgl. auch Quist (2008), für eine ausführliche Argumentation in Bezug auf Kiezdeutsch vgl. Freywald et al. (2011). 
Ein solcher Dialektbegriff bezieht damit neben horizontalen auch vertikale Bestimmungen ein, d.h. er umfasst auch Varietäten, die durch soziale Faktoren bestimmt sind. Im Fall von Kiezdeutsch kommen beide Aspekte zusammen: Kiezdeutsch ist der Sprachgebrauch bestimmter urbaner Wohngebiete und damit räumlich bestimmt, diese Wohngebiete sind jedoch, anders als herkömmliche Dialekte, nicht einer bestimmten geografischen Region zugeordnet, sondern durch einen hohen Migrantenanteil definiert, und dies ist in Deutschland, ähnlich wie in anderen westeuropäischen Ländern, mit sozialen Faktoren wie niedrigem Haushaltseinkommen und hoher Erwerbslosenquote verknüpft. ${ }^{4}$ Im Unterschied zu typischen Soziolekten ist der Gebrauch von Kiezdeutsch innerhalb dieser Wohngebiete nicht auf Sprecher/ innen einer bestimmten sozialen Schicht beschränkt, sondern wird übergreifend gesprochen.

Eine zentrale Altersgruppe für Kiezdeutsch sind Jugendliche, und auf diese Sprechergruppe werde ich mich im Folgenden in erster Linie beziehen. Wie am Beispiel von ,gibs" als Existenzmarker noch deutlich wird (Abschnitt 3.2), sind jedoch insbesondere die grammatischen Neuerungen in Kiezdeutsch (anders als die stärker jugendsprachlichen lexikalischen Erweiterungen) möglicherweise nicht nur an diese Altersgruppe gebunden. ${ }^{5}$

Der oben zitierte Dialektbegriff setzt ein charakteristisches sprachliches System voraus, mit Besonderheiten auf grammatischer, phonologischer und lexikalischer Ebene. Für Kiezdeutsch sind eine Reihe von Charakteristika beschrieben worden, die diese Bedingung erfüllen. ${ }^{6}$ Beispiele sind etwa

auf grammatischer Ebene:

- der Gebrauch bloßer Nominalphrasen als Orts- und Zeitangaben (1a) oder mit semantisch gebleichten Verben (1b): ${ }^{7}$

(1) a. gehst du heute AUCH viktoriapark?

(Wiese 2009, S. 792)

b. hast du problem?

(Auer 2003, S. 258)

$4 \quad$ Vgl. OECD International Migration Outlook 2006 (OECD Publishing, doi: 10.1787/migr_outlook -2006-en); Mikrozensus 2005 des Statistischen Bundesamtes zur Bevölkerung mit Migrationshintergrund in Deutschland (www.destatis.de/DE/Publikationen/Thematisch/Bevoelkerung/Migration Integration sowie exemplarisch den Bericht 2010 der Berliner Senatsverwaltung für Stadtentwicklung, Bereich „Monitoring Soziale Stadtentwicklung " (www.stadtentwicklung.berlin.de/ planen/basisdaten_stadtentwicklung/monitoring/de/2010/index.shtml).

5 Vgl. auch Rampton (ersch.demn.) für ähnliche Beobachtungen in Großbritannien. Vgl. Cheshire et al. (2011) zur Entstehung urbaner Multiethnolekte als eine neue Form der Dialektbildung, mit sprachlichen Merkmalen, die oft bei jugendlichen Sprecher/inne/ $\mathrm{n}$ besonders ausgeprägt, aber auch in anderen Altersgruppen zu finden sind.

- Vgl. Keim/Androutsopoulos (2000); Androutsopoulos (2001a, 2001b); Kallmeyer/Keim (2003); Auer (2003); Dirim/Auer (2004); Kern/Selting (2006a, 2000b); Tertilt (1996); Wiese (2006, 2009, 2012).

7 Für eine Analyse der Konstruktionen aus bloßen Nominalen und semantisch gebleichten Verben als produktive neue Funktionsverbgefüge in Kiezdeutsch vgl. Wiese (2006). 
- neue Möglichkeiten zur Besetzung des Vorfelds (in selbstständigen Aussagesätzen zusätzlich zur Verb-zweit-Stellung auch Verb-erst-Stellung (2a) oder mehrfache Vorfeldbesetzung (2b)):
a. wollt ich keine hektik machen
(Dirim/Auer 2004, S. 207)
b. jetz ich bin 18
(Auer 2003, S. 259)

auf phonologischer Ebene:

- die Koronalisierung des palatalen Frikativs [c] zu [६] bzw. [S];

- einige phonetische Reduktionen, etwa der Gebrauch von [s] statt [ts] in wortinitialer Position.

auf lexikalischer Ebene:

- die Integration neuer Fremdwörter aus den Herkunftssprachen unterschiedlicher Sprecher/innen, etwa „lan" im Sinne von jugendsprachlichem ,Alter" aus dem Türkischen (dort ursprünglich in der Bedeutung ,Kerl' ${ }^{\mathrm{c}}$ :

(3) isch will mit dir spielen lan

(Kallmeyer/Keim 2003, S. 33)

- die Bildung neuer Funktionswörter, etwa „lassma“ und ,musstu“ (aus „lass uns ma(l)" und ,musst $\mathrm{du}^{c}$ ) zur Markierung sprecher-inklusiver vs. -exklusiver Direktiva:

(4) a. lassma Moritzplatz aussteigen

(Wiese 2009, S. 799)

b. musstu lampe reinmachen

(Wiese 2009, S. 802)

Wie wir an anderer Stelle gezeigt haben (Wiese 2006, 2009; Freywald et al. 2011), enstehen neue grammatische Muster und Funktionswörter in Kiezdeutsch durch ein systematisches Zusammenspiel unterschiedlicher grammatischer Teilsysteme und ihre Interaktion mit außergrammatischen Domänen wie Diskurs und Informationsstruktur. Diese Systematik in der Herausbildung sprachlicher Charakteristika etabliert Kiezdeutsch als eigene Varietät des Deutschen, die dabei, ebenso wie andere Dialekte, in das sprachliche System des Deutschen eingebettet ist und sich so ins dialektale Spektrum einfügt.

Als neuer, junger Dialekt, der anders als andere heutige Dialekte des Deutschen noch nicht über eine so lange Entwicklungsgeschichte verfügt, ist Kiezdeutsch sprachwissenschaftlich besonders interessant. Der mehrsprachige Kontext gibt diesem Dialekt zudem eine besondere Dynamik. Durch die Sprachenvielfalt und die vielsprachigen Kompetenzen, die die 
multiethnische Sprechergemeinschaft auszeichnen, steht das Deutsche hier in einer Vielzahl von Sprachkontaktsituationen. Die bisher untersuchten sprachlichen Charakteristika von Kiezdeutsch weisen darauf hin, dass dies zu einer größeren Offenheit gegenüber sprachlicher Variation führt, die sprachliche Entwicklungen besonders stützt, dass diese Entwicklungen aber in erster Linie binnenstrukturell und motiviert sind und weniger durch direkte kontaktsprachliche Übertragungen. ${ }^{8}$

Die Perspektive auf Kiezdeutsch als Dialekt kann dies erfassen und damit zu einem neuen Verständnis von Kiezdeutsch beitragen. Sie erlaubt es, die reichhaltigen Erkenntnisse aus der Dialektologie des Deutschen und aus der soziolinguistischen Dialektforschung für die Interpretation unserer Befunde zu nutzen, nicht nur, was die Ebenen von Sprachsystem und Diskurs angeht, sondern, wie ich an anderer Stelle gezeigt habe (Wiese 2012, Teil 2), auch in Bezug auf perzeptuelle und sprachideologische Evidenz zu Kiezdeutsch.

Die Einordnung als Dialekt erfasst zudem ein weiteres Merkmal, das Kiezdeutsch mit herkömmlichen Dialekten des Deutschen teilt, nämlich die Gradierung seines Gebrauchs: Wie andere heutige Dialekte auch wird Kiezdeutsch in unterschiedlich starker Ausprägung gebraucht, wir finden weniger strikt kategoriale Unterschiede zu anderen Sprechergemeinschaften als vielmehr graduelle Übergänge zu anderen Varianten, etwa stärker standardnaher Sprache, und eine gezielte Wahl aus einem Repertoire von Merkmalen je nach Sprechsituation. Dies ist ein generelles Phänomen im heutigen Dialektgebrauch; die Charakterisierung von Kiezdeutsch als Dialekt ist daher besonders geeignet, die Einordnung dieser Sprechweise als „Stil", die dies aufgreift, mit der stärker grammatiktheoretischen Einordnung als ,Varietät" zu verknüpfen.?

\section{Grammatische Entwicklungstendenzen im mehrsprachigen Kontext: zwei Beispiele}

Generell lassen sich zwei Arten grammatischer Entwicklungen in Kiezdeutsch identifizieren: (1) der quantitative Ausbau von NichtstandardKonstruktionen gegenüber dem Sprachgebrauch in vergleichbaren stärker monoethnischen/monolingualen Sprechergemeinschaften, d.h. von Konstruktionen, die in anderen Varietäten des Deutschen ebenfalls auftreten, dort aber nicht so stark verbreitet oder weniger systematisch etabliert sind; (2) grammatische Innovationen, die auf vorhandenen Mustern des Deutschen aufbauen, diese aber qualitativ verändern und weiterentwickeln. Im

\footnotetext{
8 Vgl. auch Poplack/Levey (2010) generell zur Differenzierung kontaktsprachlicher Einflüsse und internen Wandels.

Vgl. auch oben Fußnote 3.
} 
Folgenden werde ich für die beiden Bereiche je ein Beispiel genauer darstellen: (1) die quantitative Expansion von intensivierend gebrauchtem „,voll ${ }^{\text {"c }}$, das ebenso auch aus anderen Varianten des Deutschen bekannt ist, und (2) der Gebrauch von ,gib(t)s" als monomorphematische Existenzpartikel. Der Fall von ,gib(t)s" illustriert zudem, wie Entwicklungstendenzen im mehrsprachigen Kontext verstärkt werden können, indem die binnenstrukturelle, im sprachlichen System des Deutschen angelegte, Motivation kontaktsprachlich noch weiter gestützt wird, in diesem Fall durch das Vorliegen einer ähnlichen Existenzpartikel im Türkischen.

\subsection{Quantitative Expansion: intensivierendes „,voll ${ }^{\text {“ }}$}

„voll“" ist in seiner herkömmlichen Verwendung eine Präposition (,eine Flasche voll Wein/voller Wein") oder ein Adjektiv („eine volle Flasche"), das sich semantisch auf das Volumen von Behältern bezieht und ein Gegen-

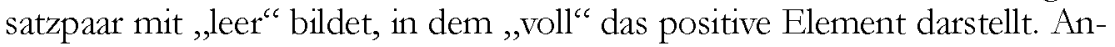
ders als ein positiv gerichtetes Dimensionsadjektiv wird „,voll“ nicht mit Maßangaben gebraucht (,,4kg schwer" vs. *, ,4 Liter voll"), sondern gibt ein Maximum an und gehört in dieser Hinsicht in ein semantisches Feld mit Adjektiven wie ,ganz" oder ,total". ${ }^{10}$ Ähnlich wie diese wird ,voll" in informeller Sprache intensivierend gebraucht und steht damit in ähnlicher Distribution wie ,sehr":

$$
\text { Das ist }\{\text { voll/ganz/total/sehr }\} \text { schön. }
$$

„,voll“ kann hier semantisch, ähnlich wie „sehr“, Eigenschaften ebenso wie Handlungen modifizieren und syntaktisch entsprechend sowohl mit Adjektiv- und Adverbphrasen als auch mit Verbphrasen stehen. Diese Verwendung von ,voll“" ist besonders in Jugendsprache verbreitet und hier so salient, dass sie mindestens seit den 1980ern in Lexika der Jugendsprache Eingang gefunden hat, dies sowohl im ostdeutschen als auch im westdeutschen Raum. $^{11}$

(6) gibt einige Beispiele aus dem KiezDeutsch-Korpus („KiDKo einem Korpus spontansprachlicher Daten aus Gesprächen Jugendlicher, das auf Eigenaufnahmen in Peer-Group-Situationen basiert (vgl. Wiese et al. 2012). Das KiDKo umfasst ein Hauptkorpus von rund 48 Aufnahmestunden (ca. 228.000 Token; 17 Anker-Sprecher/innen) mit Daten ein- und mehrsprachiger Sprecher/innen aus einem multiethnischen Wohngebiet, BerlinKreuzberg, und ein Ergänzungskorpus von rund 18 Aufnahmestunden

\footnotetext{
10 Kirschbaum (2002, Kap. 2.2.2) charakterisiert Intensivierer wie „,voll“" entsprechend als (Skalen-)Endpunktadjektive. Vgl. auch Kirschbaum (ebd., Kap. 5.8.2) zum metaphorischen Aus-

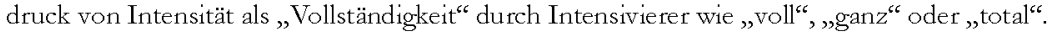

11 Vgl. exemplarisch Müller-Thurgau (1985), Heinemann (1989).
} 
(ca. 105.000 Token; 6 Anker-Sprecher/innen) mit Daten aus einem sozioökonomisch vergleichbaren, aber weitgehend monoethnischen Wohngebiet, Berlin-Hellersdorf.

Die Verwendung von intensivierendem ,,voll " fand sich sowohl in der multiethnischen als auch in der monoethnischen Population (die unten angegebenen Sigel geben mit den ersten beiden Buchstaben die Zugehörigkeit der Daten zur multiethnischen $(\mathrm{Mu})$ bzw. monoethnischen (Mo) Population an; die letzten beiden Buchstaben verweisen auf das Geschlecht der Ankersprecher/innen $(\mathrm{M} / \mathrm{W})$ und ihre Familiensprache, d.h. die Sprache, die in der Familie hauptsächlich verwendet wird, mit ,D" für Deutsch, „T" $T^{c c}$ für Türkisch, „K“ für Kurdisch und „A“ für Arabisch; Versalien markieren Hauptakzente):

(6) a. oh ich bin heut morgen voll LUStig, (KiDKo, MuH11MD)

b. es hat nisch weHgetan, aber es is voll FETT gworden . (--) sieht voll SCHLIMM aus (KiDKo, MuH19W'T)

c. dass sie ihn voll süss findet. (KiDKo, Mo01MD)

d. das habt ihr aber wIRKlich voll oft ne ? (KiDKo, MuH1WD)

e. isch zITter immer voll und dann verRUTSCHT es jedes mal. (KiDKo, MuH19WT)

f. musst ick erstma voll LAChen. (KiDKo, Mo18MD)

Neben herkömmlichen Adjektiven wie in $(6 a-c)$ treten als Kopf der modifizierten Phrase auch solche auf, die aus evaluativen Nomen hervorgegangen sind; vgl. (7): ${ }^{12}$
a. ah mann dis sieht man voll scHEIße so. (KiDKo, MuH11MD)
b. dis is voll KILler man . (KiDKo, MuH HWT)
c. das is doch voll SCHROTT. (KiDKo, MuH12MD)

Eine weitere intensivierende Verwendung von „,voll“ ist die am linken Rand definiter DPs, ${ }^{13}$ wie sie in (8) durch drei Korpusbeispiele illustriert ist:

\footnotetext{
12 Vgl. Androutsopoulos (1998) zu diesem Muster desubstantivischer Konversion in Jugendsprache. Pittner/Berman (2006) gehen davon aus, dass das Auftreten in prädikativer Position, wie es in (b) und (c) deutlich wird, die Basis für die Entwicklung darstellt.

13 Für eine ausführliche Analyse der Syntax dieser Konstruktion vgl. Meinunger (2009).
} 


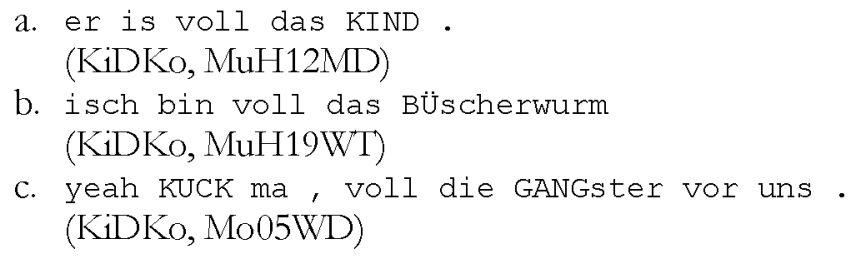

Diese Verwendung ist sowohl syntaktisch als auch semantisch interessant. Auf syntaktischer Ebene fällt die Kombination mit adjazent nachfolgendem Definitartikel mit lexikalischer Nominalphrase/NP auf, ohne Genitivzuweisung von „,voll“, anders als etwa in herkömmlichem, veraltendem Gebrauch von ,voll ${ }^{c}$ als Präposition mit einer Determinansphrase/DP als Komplement (,voll des Weines"). Trotz des definiten Artikels verhält sich die Konstruktion nicht semantisch definit. ${ }^{14}$ Sie ist dennoch semantisch nicht äquivalent zu indefiniten Konstruktionen, wie dies Androutsopoulos (1998) etwa nahelegt, ${ }^{15}$ sondern wird, anders als diese, häufig für einen Prototypen-Bezug gebraucht. ${ }^{16}$

Der semantische Beitrag von Definitartikel und NP kann in diesem Fall als Identifizierung eines prototypischen Exemplars beschrieben werden, während ,voll“ die vollständige, maximale Subsumtion unter diesen Typus betont: Es geht hier um die Erfïllung aller Merkmale des Prototyps. So bedeutet beispielsweise ,Sie ist voll die Siegerin. " nicht einfach , Sie hat gesiegt.". Eine passende Paraphrase wäre vielmehr so etwas wie ,Sie hat alle wesentlichen Merkmale einer typischen Siegerin.". Der Prototyp, um den es bei der Konstruktion mit ,,voll " geht, rekurriert begrifflich auf die gesamte NP und führt daher zu Interpretationsunterschieden in Minimalpaaren wie (9a) vs. (9b):

(9) a. ein voll schöner Mann

b. voll der schöne Mann

In (a) modifiziert ,voll“" als Intensivierungspartikel die Adjektivphrase/AP „schöner“; es geht um einen Mann, der besonders schön ist. In (b) bezieht sich ,,voll ${ }^{c c}$ dagegen nicht auf schön allein, sondern auf ,schöner Mann ${ }^{c}$; hier geht es um jemanden, der alle Merkmale eines prototypischen schönen Mannes besitzt, d.h. jemand, der den Prototyp des „schönen Mannes“ instantiiert ((9b) könnte daher auch negativ wertend im Sinne von ,ein Schönling" gebraucht werden).

\footnotetext{
14 Etwa *,Dort sind voll die Gangster, die ich vorhin gesehen habe.“; vgl. Gutzmann/Turgay (2011) (ähnlich auch für intensiverendes totalund sau).

15 Vgl. Androutsopoulos (1998, S. 355), der zu Varianten wie ,eine sehr schöne Frau“ und ,voll die schöne Frau“ feststellt: „,Die Varianten unterscheiden sich in Wortstellung [...] und Artikelwahl [...], sind aber semantisch-funktional äquivalent, da sich weder die Funktionsbedeutung der Intensivierung noch die deskriptive Bedeutung der intensivierten Phrase ändert. ${ }^{c c}$

16 Vgl. auch Meinunger (2009).
} 
Syntaktisch weist die Konstruktion ,voll Definitartikel NP" Parallelen zu ,ganz der Vater" auf. In der Konstruktion mit ,ganz" bezieht sich die definite DP jedoch regulär auf das einzige/salienteste/maximale Element, das die NP begrifflich instantiiert, nicht auf einen Prototyp. (10) illustriert dies an Paraphrasen für ein (konstruiertes) Minimalpaar mit , $\{$ gan $/$ voll $\}$ Def.art. $\mathrm{NP}^{\varsigma}$ :

a. Er ist ganz der Vater.

$\rightarrow$,Er hat alle wesentlichen Merkmale seines (= des spezifischen, kontextuell salienten) Vaters.'

b. Er ist voll der Vater.

$\rightarrow$,Er hat alle wesentlichen Merkmale eines typischen Vaters. ${ }^{c}$

Um die Distribution und den Ausbau der Nichtstandard-Verwendung von "voll" in intensivierender Bedeutung in multiethnischen Sprechergemeinschaften zu untersuchen und mögliche Unterschiede in der Gebrauchshäufigkeit gegenüber monoethnischen Sprechergemeinschaften zu identifizieren, wurde auf der Basis des KiezDeutsch-Korpus eine Korpusstudie zum Auftreten von „,voll“ in spontansprachlichen Gesprächen Jugendlicher durchgeführt (in Haupt- und Ergänzungskorpus, d.h. für die multi- und die monoethnische Population). ${ }^{17}$

Hierzu wurden zunächst sämtliche Vorkommnisse von ,voll“" im Korpus extrahiert und manuell klassifiziert. Insgesamt ergab die Suche 1.185 Vorkommnisse von ,voll“" im Korpus oder 3,57 Verwendungen pro 1.000 Wörter. Hierbei fanden sich in beiden Teilkorpora (multi- und monoethnische Population) herkömmliche Verwendungen von „voll “ im Sinne von "gefüllt $t^{\text {" }}$ ebenso wie intensivierende Verwendungen in Modifikation von APs, AdvPs und VPs (vgl. (6) und (7) oben) und in der Konstruktion mit definiter DP (vgl. (8) oben). Die quantitative Auswertung der verschiedenen Verwendungsweisen und der Vergleich von multi- und monoethnischer Population ergab zwei interessante Befunde:

1. Dominanz der intensivierenden Verwendung: „voll" tritt, insgesamt betrachtet, dominant in intensivierender Verwendung auf, nämlich in über $95 \%$ der Fälle. Die herkömmliche Verwendung von ,voll“ im Sinne von ,gefüllt" macht demgegenüber mit knapp 5\% (54 Vorkommnisse) nur einen kleinen Teil der ,,voll“"-Vorkommnisse aus. Innerhalb dieser Gruppe fanden sich nur zwei Fälle, je einer aus Haupt- und Ergänzungskorpus, in denen „,voll ${ }^{c 6}$ als Präposition mit einer NP/DP kombiniert wurde:

\footnotetext{
17 Die Studie wurde im Frühjahr 2011 durchgeführt, Grundlage war ein Korpusumfang von 228.917 Token im multiethnischen Hauptkorpus und 103.160 Token im monoethnischen Ergänzungskorpus.
} 
a. aber du ÄRgerst sie, du machst sievoll kompLExe. (.) isch schwöre. (KiDKo, MuP05WK)

b. alles (.) is voll DRECK. (KiDKo, Mo05WD)

Der erste Beleg (11a) ist dabei keine eindeutige Instanz für ,,voll ${ }^{c \varsigma}$ als Präposition im Sinne von, gefüllt": Möglicherweise ist ,voll" hier eher als Intensivierungspartikel in der Verbphrase/VP gebraucht, das Pronomen ,sie ${ }^{c c}$ wäre dann als Akkusativ-Pendant zu standardsprachlichem Dativ zu verstehen (= Interpretation im Sinne von ,Du machst ihr \{voll/total\} Komplexe.").

Wir finden in beiden Belegen keine overte Kasuszuweisung an die NP/ DP, beide NPs sind artikellos und indefinit (Pluralnomen bzw. transnumerales Singularnomen). Zur Kombination mit NP/DP wird zudem in zwei Fällen im Hauptkorpus statt einer direkten Subordinierung eine Einbettung der NP/DP in eine PP mit ,,mit" gewählt, wie wir sie auch aus Hörbelegen in informeller Sprache kennen:

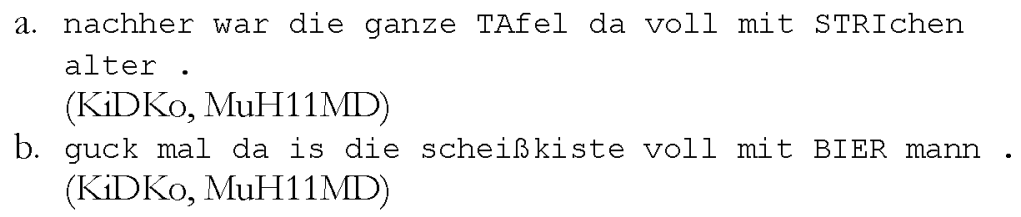

Zusammengenommen könnte dies darauf hinweisen, dass die Verwendung von ,voll“" als Präposition im Rückgang begriffen ist. Dies wäre allerdings noch durch diachrone Vergleichsdaten zu belegen; möglich wäre auch ein generell marginales Auftreten dieser Konstruktion.

2. Dominanz in der multiethnischen Population: ,voll" wird in der multiethnischen Population mehr als doppelt so oft gebraucht wie in der monoethnischen Population, nämlich 4,25 Mal pro 1.000 Wörtern im Hauptkorpus gegenüber 2,06 Mal pro 1.000 Wörtern im Ergänzungskorpus. Diese Dominanz geht auf eine häufigere intensivierende Verwendung von ,,voll“" zurück, nämlich 4,1 Mal pro 1.000 Wörtern im Hauptkorpus ( $m i$ ) gegenüber 1,87 Mal pro 1.000 Wörtern im Ergänzungskorpus (mo), und dies betrifft sowohl die Verwendung als Intensivierungspartikel in APs und VPs (mu: 4,10; mo: 1,87) als auch die in "Prototyp"-Konstruktionen der Form , , wll Defart. $\mathrm{NP}^{c}$ (mu: 0,30; mo: 0,16). Im Gegensatz dazu kommt ,,voll ${ }^{c c}$ in herkömmlicher Verwendung ( $=$ in der Bedeutung ,gefüllt $\left.t^{\circ}\right)$ in beiden Teillkorpora fast gleich oft pro 1.000 Wörtern vor, in der monoethnischen Population sogar etwas häufiger, nämlich 0,18 Mal, gegenüber 0,15 Mal im Hauptkorpus (bzw. bei rund 9\% aller ,voll ${ }^{\varsigma}$-Vorkommnisse im Ergänzungskorpus und bei rund $4 \%$ aller ,,voll"s-Vorkommnisse im Hauptkorpus). 
Zusammengenommen lässt sich damit festhalten: „voll “ tritt nach diesen Korpusbefunden in gesprochener informeller Sprache unter Jugendlichen dominant in Nichtstandard-Verwendung auf, nämlich in intensivierender Funktion einschließlich der in „Prototy $\mathrm{p}^{c}$-Konstruktionen der Form „voll Def.art. NP" der multiethnischen Population des Hauptkorpus noch einmal bedeutend weiter verbreitet als in der monoethnischen Population des Ergänzungskorpus. Die multiethnische Sprechergemeinschaft erweist sich damit als ein Kontext, in dem sprachliche Entwicklungen in informeller Sprache besonders deutlich werden.

\subsection{Ausbau und Innovation: monomorphematisches, gib $(t) s^{c c}$}

Für existenzanzeigendes „geben“ finden wir im Kontext mehrsprachiger Sprechergemeinschaften eine interessante Entwicklung zu einem monomorphematischen Element, das Merkmale einer Existenzpartikel aufweist.

Im Vergleich zu ,geben" im Sinne von ,überreichen" ist existenzanzeigendes ,geben" im Deutschen in seinem Konstituentenrahmen reduziert und stärker spezifiziert. Auf semantischer Ebene vergibt es nur eine thematische Rolle, das Thema (= die Entität, für die Existenz prädiziert wird), die auf syntaktischer Ebene mit dem Akkusativobjekt assoziiert ist, während als Subjekt ein semantisch leeres Expletivum „es" auftritt. ${ }^{18}$ Die Konstruktion ist daher in zweifacher Hinsicht auffallig: Erstens gibt es ein syntaktisches Argument, das Subjekt, das kein semantisches Pendant hat, und umgekehrt ist die höchste thematische Rolle, hier das Thema, nicht mit dem Subjekt assoziiert, sondern mit dem Objekt. Existenzanzeigendes ,geben ${ }^{c}$ weicht damit von der regulären Organisation verbaler Argumentstrukturen ab. Zweitens ist das Verb in dieser Konstruktion durch das feste Subjekt „es" auf die 3. Person Singular (in finiten Vorkommnissen) beschränkt. Existenzanzeigendes ,geben " ist damit in seinem morphosyntaktischen Paradigma gegenüber regulären Verbformen eingeschränkt.

Der zweite Punkt stützt eine Entwicklung zu einer monomorphematischen Form ,gib(t)s" in der gesprochenen und/oder konzeptionell mündlichen Sprache auch außerhalb von Kiezdeutsch. Existenzaussagen werden typischerweise nicht global getroffen, sondern auf einen spezifischen Rahmen eingeschränkt (vgl. Lambrecht 1995). Da Rahmensetzer generell an den linken Rand der Satzperipherie streben (vgl. Jacobs 2001; Krifka 2007),

18 Lenerz (1992) nennt dies ein „Subjekt-es“, im Gegensatz zum „Topik-es", das kein obligatorisches Element des Verbs ist, sondern zusätzlich zu einem vollen Subjekt gebraucht wird, in der linken Peripherie basisgeneriert wird und nicht in der Basisposition des Subjekts erscheinen kann. Czinglat (1997) betont, dass das Expletivum bei existenzanzeigendem "geben“ immer die Form „es" hat, im Gegensatz etwa zu Wetterverben, die auch „das" als Subjekt zulassen. 
steht das Subjekt von existenzanzeigendem ,geben " meist nicht im Vorfeld, sondern in seiner Basisposition nach dem finiten Verb. Dies liefert eine Linearisierung, bei der im Indikativ Präsens typischerweise ,gibt" von „es" gefolgt wird, das als schwaches Pronomen in gesprochener Sprache dann klitisiert wird, d.h. finden wir meist , gibt's" bzw. die noch weiter reduzierte Form , gibs“" ${ }^{19}$

Die Häufigkeit dieser Form scheint auch außerhalb von Kiezdeutsch die Uminterpretation als monomorphematisches Element zu stützen, bei dem ,(e)s" dann nicht mehr als klitisiertes Subjekt zugänglich ist. Einen Hinweis hierauf liefern Daten aus konzeptionell mündlicher Sprache, in denen ein zusätzliches volles Subjekt ,es" zu existenzanzeigendem, gibts" hinzukommt. (13) gibt drei Beispiele aus Internet-Diskusssionen:
a. Es gibts nicht zum Anziehen für den Schulanfang! ${ }^{20}$
b. Es gibts Jobs ... um die beneide ich keinen. ${ }^{21}$
c. Es gibts nichts besseres!! Einfach Wunderschön ${ }^{22}$

Vergleichbare Konstruktionen finden sich auch im Kontext mehrsprachiger Sprechergemeinschaften, vgl. (14) (aus einem Interview-Transkript, türkischdeutscher Sprecher aus Berlin-Kreuzberg):

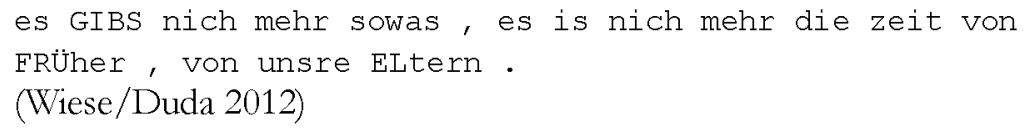

Diese Univerbierung von, ,gibts" liefert die Basis für eine Weiterentwicklung in Kiezdeutsch, die die grammatische Dynamik aufnimmt, die sich aus der oben angesprochenen irregulären Argumentstruktur speist, und eine Alignierung syntaktischer und semantischer Argumente und damit eine Regularisierung der Konstruktion bewirkt. Die monomorphematische Form „gib(t)sc wird nach diesem weiteren Entwicklungsschritt nicht mehr mit einem Expletivum kombiniert, sondern hat als einziges Argument das Thema. Beispiele hierfür finden sich sowohl im KiezDeutsch-Korpus (vgl. (15a), (15b)) als auch in Hörbelegen von älteren Sprecher/inne/n (vgl. (15c)-(15e); sämtlich türkisch-deutsche Sprecher/innen im Alter von 30 bis 40 Jahren in Berlin-Kreuzberg) und von Vorschulkindern ((15f)-(15h); aus einer Studie

\footnotetext{
Im DWDS-Kernkorpus finden sich beispielsweise 13.000 Vorkommnisse von „es gibt“

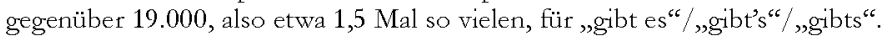

20 Internetforum zu „Kids und Schule“, www.urbia.de/archiv/forum/th-2175813/Es-gibts-nicht-zum -Anziehen-fuer-den-Schulanfang.html (Stand: 29.02.2012).

21 Leserkommentar zu Artikel über neuen Finanzminister in Griechenland in Focus Online vom 17.06.2011, www.focus.de/politik/ausland/es-gibts-jobs-griechenland-kommentar_3591886.html (Stand: 29.02.2012).

22 Bewertung eines Hotels durch Reisende auf TripAdvisor, www.tripadvisor.de/ShowUserReviews -g194907-d277291-r122003302-Grand_Hotel_la_Pace-Sant_Agnello_Province_of_Naples_Campania.html (Stand: 29.02.2012).
} 
in Kreuzberger Kindergärten; vgl. Wiese/Duda 2012; zu den Sprecher/innen im Einzelnen: (15f) - 6 Jahre alt, englisch-deutsch; (15g) - 5 Jahre alt, türkisch-deutsch; (15h) - 5 Jahre alt, einsprachig deutsch):
a. GUCK ma was hier alles NOCH gibs . (KiDKo, MuP1MK)
b. WEIßte doch, die die in verschiedene FARben gibs ? (KiDKo, MuH9WT)
c. ich such mal RAUS wo das gibs.
d. ich war gestern BAUhaus und habe geguckt welche sorten gibs.
e. ich gucke mal nach ob auch bezüge für KINdersitze gibs.
f. ich WEIß wo die gibs.
g. hast du vergessen dass auch SCHLUMPFeis gibs ?
h. aber den BABYcarrier weiß ich nicht wo den gibs. den hat mir meine Oma geschenkt.

Die Wortstellung deutet hier darauf hin, dass, gibs“ als Partikel verwendet wird, die die Position des finiten Verbs einnimmt. Diese Konstruktion ist an der Oberfläche nur bei dieser Wortstellung, in subordinierten Sätzen, von der herkömmlichen Konstruktion mit existenzanzeigendem finitem Verb und klitisiertem Subjekt unterscheidbar (vgl. etwa „Die gibs in verschiedene Farben." bzw. „Gibs die in verschiedene Farben?"). Diese Parallelen könnten die Uminterpretation von ,gib $(t) s^{c}$ stützen. Ein zweiter Punkt betrifft den syntaktischen Status des nunmehr einzigen Arguments, des Themas: Wegen des weitgehenden Formen-Synkretismus in Nominativ und Akkusativ ist oft nicht sichtbar, ob es sich jeweils um das Objekt oder das Subjekt handelt. Diese Ambiguität an der Oberfläche könnte eine Uminterpretation des ursprünglichen Objekts von existenzanzeigendem ,geben“ als - meist formgleiches - Subjekt begünstigen. Die Kreuzberger Kinderdaten weisen an einigen Stellen darauf hin, dass dies ein möglicher weiterer Entwicklungspfad sein könnte: In Fällen, in denen maskuline Singularformen auftreten, die auch oberflächlich eine Kasus-Unterscheidung erlauben, finden sich neben Akkusativformen auch einige eindeutige Nominative, vgl. etwa die Beispiele in (16) (Sprecher/innen: (16a) - 5 Jahre alt, türkisch-deutsch; (16b) - 4 Jahre alt, einsprachig deutsch; (16c) - 5 Jahre alt, türkisch-deutsch):

(16) a. welche ninjas SIND denn hier ? zeig mir mal WER alles gibs.

b. ich mag BEIde sorten pudding . das is gut weil wenns nur EIner gibs, mag ich den auf JEden fall.

c. das is kein geschmack von einer FRUCHT . das is einfach ein geschmack, der woanders gar nicht GIBS . 
Dies weist auf eine mögliche Weiterentwicklung von ,gib(t) $\mathrm{s}^{\text {c }}$ in mehrsprachigen Sprechergemeinschaften, die in letzter Konsequenz zu einer Regularisierung gegenüber der herkömmlichen, devianten Konstruktion in Bezug auf die Syntax-Semantik-Alignierung führen kann: Es findet sich kein syntaktisches Argument ohne semantisches Gegenstück mehr („es"), und die höchste thematische Rolle (das Thema) korrespondiert mit dem Subjekt, nicht mit dem Objekt. ${ }^{23}$ Ein neues Spannungsfeld entsteht für die Konstruktion demgegenüber auf morphosyntaktischer Ebene: Die Form ,gib(t)s $\mathrm{s}^{c}$, die nun an der Position des finiten Verbs steht, ist hier (noch?) eine Partikel, die keine Flexionsmerkmale aufweist und entsprechend auch nicht mit dem neuen Subjekt kongruieren kann. Wenn sich die Entwicklung, die sich hier für , gib $(t) s^{c}$ in mehrsprachigen Sprechergemeinschaften andeutet, weiter konsolidiert, wäre eine Fortführung denkbar, in der dann entsprechende finite Formen entstehen.

Die Entwicklung von ,gib(t)s" zu einem monomorphematischen Existenzanzeiger passt, wie hier deutlich wurde, grundsätzlich ins System des Deutschen; sie ist binnenstrukturell gut motiviert und tritt, wie etwa (15h) und (16b) illustrieren, auch bei einsprachig deutschen Kindern in multiethnischen Sprechergemeinschaften auf. Die neue Verwendung von, gib $(t) s^{c /}$ in solchen Sprechergemeinschaften könnte jedoch zusätzlich noch kontaktsprachlich untermauert werden, nämlich durch das Türkische, das mit dem Existenzanzeiger „var" (verneint: ,yok ${ }^{c}$ ) ein ganz ähnliches Element besitzt. Ein möglicher kontaktsprachlicher Einfluss ist hier nicht so sehr in Form von Interferenzen plausibel, die jeweils in der konkreten Äußerung aktiviert würden und nur Sprecher/innen mit Türkischkompetenzen betreffen könnten, sondern eher in Form einer weiteren Stützung. Da ein relativ großer Anteil der Sprecher/innen das Türkische beherrscht und somit die Verwendung einer Existenzpartikel im sprachlichen Repertoire hat, ist vorstellbar, dass diese Sprecher/innen gegenüber der Entwicklung von, gib(t)s“ zur Existenzpartikel besonders offen sind; diese Offenheit könnte die Verbreitung in der Sprechergemeinschaft dann insgesamt noch begünstigen.

\section{Fazit und Ausblick}

Der vorliegenden Beitrag hat den Sprachgebrauch in multiethnischen und multilingualen Sprechergemeinschaften in Deutschland aus der Perspektive grammatischer Entwicklungen untersucht. Hierbei konnten zwei zentrale Entwicklungspfade identifiziert werden: die quantitative Expansion von Konstruktionen, die auch aus anderen Varietäten des Deutschen bekannt

23 Für eine formale Modellierung der Syntax-Semantik-Korrespondenzen vgl. Wiese/Duda (2012). 
sind, und grammatische Neuerungen, die vorhandene Entwicklungstendenzen des Deutschen aufnehmen und qualitativ erweitern. Wie die hier vorgestellten exemplarischen Analysen für die beiden Domänen illustrierten, sind diese Entwicklungen, auch im Fall grammatischer Neuerungen, wesentlich systemintern, binnenstrukturell im Deutschen motiviert. Der hohe Anteil mehrsprachiger Sprecher/innen in multiethnischen Sprechergemeinschaften trägt dabei zu einer besonderen Dynamik der hier entstehenden urbanen Dialekte bei, und dies, wie ich argumentiert habe, auf zwei Ebenen: Zum einen generell durch eine größere Offenheit gegenüber sprachlicher Variation, die Prozesse von Sprachwandel und Sprachentwicklung besonders begünstigt; zum anderen durch Sprachkontaktinstanzen, die in konkreten Fällen Entwicklungen, die im System des Deutschen angelegt sind, noch verstärken können.

Multiethnische Sprechergemeinschaften weisen damit ein besonderes Potenzial für Sprachwandel und grammatische Innovation auf. Sie liefern den Kontext für urbane Dialekte, in denen Entwicklungstendenzen im Gegenwartsdeutschen besonders deutlich werden.

Dies steht in auffälliger Diskrepanz zur Wahrnehmung dieser Sprechergemeinschaften und ihres Sprachgebrauchs in der öffentlichen Diskussion (vgl. Wiese 2010, 2012). Ein urbaner Dialekt wie Kiezdeutsch löst hier massive Sprachkritik aus, die sich in das generelle Topos der „Sorge um den Sprachverfall ${ }^{c c}$ einfügt ${ }^{24}$ dabei jedoch noch verstärkt wird durch einen verbreiteten sprachideologischen Konnex zwischen „Migrationshintergrund"

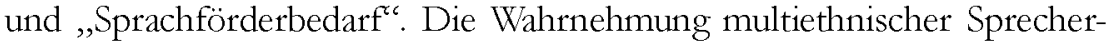
gemeinschaften ist dabei primär alloethnisch fokussiert.

Vor diesem Hintergrund werden sprachliche Neuerungen nicht als Beispiele für Variation im Gegenwartsdeutschen angesehen, sondern als Hinweis auf mangelnden Fremdspracherwerb, sprachliche Defizite und/oder massive grammatische Interferenzen aus verschiedenen Herkunftssprachen. ${ }^{25}$ Ein genauerer Blick auf die sprachlichen Phänomene offenbart dagegen, wie ich in diesem Beitrag illustriert habe, eine grundlegende Verankerung im System des Deutschen und eine sprachliche Dynamik multiethnischer, ausgeprägt mehrsprachiger Sprechergemeinschaften, die auf ein besonderes Potenzial zur Ausbildung neuer Varietäten der Majoritätssprache hinweist.

\footnotetext{
Vgl. hierzu etwa die Beiträge in Denkler et al. (Hg.) (2008).

Diese Auffassung scheint in der öffentlichen Debatte z.T. auch von linguistischer Seite vertreten zu werden, vgl. etwa Hinrichs (2012): „Das mehrsprachige Milieu kann auf korrekte Deklinationen und genaue Endungen durchaus verzichten, weil diese Art Grammatik nur Kodierungsenergie frisst, die woanders viel dringender gebraucht wird, beispielsweise um Defizite im Wortschatz auszugleichen. [...] Einwanderer greifen auch auf Sprachstrukturen zurück, die sie aus ihrer Muttersprache mitbringen. Diese werden ins Deutsche kopiert und im zweisprachigen Milieu gefestigt. Im großstädtischen Kiezdeutsch [...] gibt es etwa eine Vielzahl von Satzmustern, die aus dem Arabischen oder Türkischen stammen.“
} 


\section{Literatur}

Androutsopoulos, Jannis (1998): Deutsche Jugendsprache. Untersuchungen zu ihren Strukturen und Funktionen. (= VarioLingua 6). Frankfurt a.M. u.a.

Androutsopoulos, Jannis (2001a): From the streets to the screens and back again: On the mediated diffusion of ethnolectal patterns in contemporary German. (= LAUD Linguistic Agency: Series A, 522). Essen.

Androutsopoulos, Jannis (2001b): Ultra korregd Alder! Zur medialen Stilisierung und Aneignung von "Türkendeutsch ${ }^{c c}$. In: Deutsche Sprache 29, S. 321-339.

Auer, Peter (2003): ,Türkenslang؛: Ein jugendsprachlicher Ethnolekt des Deutschen und seine Transformationen. In: Häcki Buhofer, Annelies (Hg): Spracherwerb und Lebensalter. (= Basler Studien zur deutschen Sprache und Literatur 83). Tübingen, S. $255-264$.

Chambers, Jack K./Trudgill, Peter (1998): Dialectology. 2. Aufl. Cambridge.

Cheshire, Jenny et al. (2011): Contact, the feature pool and the speech community: The emergence of Multicultural London English. In: Journal of Sociolinguistics 15, S. 151-196.

Czinglar, Christine (1997): Bemerkungen zur Existenzbehauptung und Ortsbestimmung im Deutschen und alemannischen Varianten. In: Groninger Arbeiten zur germanistischen Linguistik 41, S. 39-60.

Denkler, Markus et al. (Hg.) (2008): frischwärts und unkaputtbar - Sprachwandel oder Sprachverfall im Deutschen. Münster.

Dirim, İnci/Auer, Peter (2004): Türkisch sprechen nicht nur die Türken. Über die Unschärfebeziehung zwischen Sprache und Ethnie in Deutschland. (= Linguistik Impulse und Tendenzen 4). Berlin/New York.

Freywald, Ulrike et al. (2011): Kiezdeutsch as a multiethnolect. In: Kern, Friederike/ Selting, Margret (Hg): Ethnic Styles of Speaking in European Metropolitan Areas. (= Studies in Language Variation 8). Amsterdam/Philadelphia, S. 45-73.

Gutzmann, Daniel/Turgay, Katharina (2011): Zur Syntax expressiver Intensivierung in der Jugendsprache. Vortrag auf der 6. Internationalen Konferenz zur Jugendsprache, „Jugendsprachen - Dynamiken und kulturelle Kontexte“, Freiburg i.Br., 31.3.-2.4.2011.

Heinemann, Margot (1989): Kleines Wörterbuch der Jugendsprache. Leipzig.

Hinrichs, Uwe (2012): Hab isch gesehen mein Kumpel. Wie die Migration die deutsche Sprache verändert hat. In: Der Spiegel 7/2012, S. 104-105.

Jacobs, Joachim (2001): The dimensions of topic-comment. In: Linguistics 39, S. 641681.

Kallmeyer, Werner/Keim, Inken (2003): Linguistic variation and the construction of social identity in a German-Turkish setting. In: Androutsopoulos, Jannis ( $\mathrm{Hg}$.): Discourse Constructions of Youth Identities. (= Pragmatics and beyond: New Series 110). Amsterdam/Philadelphia, S. 29-46. 
Keim, Inken/Androutsopoulos, Jannis (2000): Hey Lan, isch geb dir konkret Handy. Deutsch-türkische Mischsprache und Deutsch mit ausländischem Akzent: Wie Sprechweisen der Straße durch die Medien populär werden. In: Frankfurter Allgemeine Zeitung 21, 26.01.2000.

Kern, Friederike/Selting, Margret (2006a): Einheitenkonstruktion im Türkendeutschen: Grammatische und prosodische Aspekte. In: Zeitschrift für Sprachwissenschaft 25, S. 239-272.

Kern, Friederike/Selting, Margret (2006b): Konstruktionen mit Nachstellungen im Türkendeutschen. In: Deppermann, Amulf/Fiehler, Reinhard/Spranz-Fogasy, Thomas $(\mathrm{Hg})$ : Grammatik und Interaktion. Untersuchungen zum Zusammenhang von grammatischen Strukturen und Gesprächsprozessen. Radolfzell, S. 319-347.

Kirschbaum, Ilja (2002). Schrecklich nett und voll verrückt. Muster der AdjektivIntensivierung im Deutschen. Dissertation. Düsseldorf.

Krifka, Manfred (2007): Basic notions of information structure. In: Féry, Caroline/ Fanselow, Gisbert/Krifka, Manfred (Hg): The notions of information structure. (= Interdisciplinary Studies on Information Structure 6). Potsdam, S. 13-55.

Lambrecht, Knud (1995): Information structure and sentence form. Topic, focus, and the mental representations of discourse referents. (= Cambridge Studies in Linguistics ; 71). Cambridge.

Lenerz, Jürgen (1992): Zur Theorie des syntaktischen Wandels: Das expletive es in der Geschichte des Deutschen. In: Abraham, Werner (Hg.): Erklärende Syntax des Deutschen. (= Studien zur deutschen Grammatik 25). 2., überarb. Aufl. Tübingen, S. 99-136.

Meinunger, André (2009): Leftmost peripheral adverbs and adjectives in German. In: Journal for Comparative Germanic Linguistics 12, S. 115-135.

Müller-Thurgau, Claus Peter (1985): Lexikon der Jugendsprache. Düsseldorf/Wien.

Pittner, Karin/Berman, Judith (2006): video ist echt schrott aber single ist hammer - Jugendsprachliche Nomen-Adjektiv-Konversion in der Prädikativposition. In: Deutsche Sprache 34, S. 233-250.

Poplack, Shana/Levey, Stephen (2010): Contact-induced grammatical change: a cautionary tale. In: Auer, Peter/Schmidt, Jürgen Erich (Hg.): Language and Space. An International Handbook of Linguistic Variation. (= Handbücher zur Sprach- und Kommunikationswissenschaft 30). Berlin/New York, S. 391-419.

Quist, Pia (2008): Sociolinguistic approaches to multiethnolect: language variety and stylistic practice. In: International Journal of Bilingualism 12, S. 43-61.

Rampton, Ben (ersch.demn.): Contemporary urban vernaculars. In: Nortier, Jacomine/ Bente A. Svendsen ( $\mathrm{Hg}$.): Language, Youth, and Identity in the $21^{\text {st }}$ Century. Oxford.

Schroeder, Christoph (2007): Integration und Sprache. In: Aus Politik und Zeitgeschichte 22-23, S. 6-12.

Tertilt, Hermann (1996): Turkish Power Boys. Ethnographie einer Jugendbande. Frankfurt a.M. 
Trudgill, Peter (1992): Introducing language and society. London.

Wiese, Heike (2006): „Ich mach dich Messer“ - Grammatische Produktivität in KiezSprache. In: Linguistische Berichte 207, S. 245-273.

Wiese, Heike (2009): Grammatical innovation in multiethnic urban Europe: new linguistic practices among adolescents. In: Lingua 119, S. 782-806.

Wiese, Heike (2010): Führt Mehrsprachigkeit zum Sprachverfall? Populäre Mythen vom ,gebrochenen Deutsch' bis zur ,doppelten Halbsprachigkeit ${ }^{c}$ türkischstämmiger Jugendlicher in Deutschland. In: Ozil, Şeyda/Hofmann, Michael/Dayıoğlu-Yücel, Yasemin (Hg): Türkisch-deutscher Kulturkontakt und Kulturtransfer. Kontroversen und Lernprozesse. (= Türkisch-deutsche Studien 1). Göttingen, S. 73-84.

Wiese, Heike (2012): Kiezdeutsch. Ein neuer Dialekt entsteht. München.

Wiese, Heike/Duda, Sibylle (2012): A new German particle, gib(t) $s^{c}-$ The dynamics of a successful cooperation. In: Spalek, Katharina/Domke, Juliane ( $\mathrm{Hg}$.): Sprachliche Variationen, Varietäten und Kontexte. Beiträge zu psycholinguistischen Schnittstellen. (= Stauffenburg Festschriften). Tübingen.

Wiese, Heike et al. (2012): Das KiezDeutsch-Korpus. Spontansprachliche Daten Jugendlicher aus urbanen Wohngebieten. In: Deutsche Sprache 40, S. 97-123.

\section{Danksagung}

Die Arbeit an diesem Artikel wurde unterstützt durch eine Förderung der Deutschen Forschungsgemeinschaft für den Sonderforschungsbereich 632 „Informationsstruktur ${ }^{\text {cc }}$ der Universität Potsdam, der Humboldt-Universität zu Berlin und der Freien Universität Berlin, Teilprojekte B6 „Kiezdeutsch” und T1 „Urbane Sprachvariation“ (Projektleitung: H. Wiese). Für die Diskussion des hier entwickelten Ansatzes bedanke ich mich bei Teilnehmer/inne/n der DGfS-Jahrestagung 2011, der IDS-Jahrestagung 2012, des Berkeley Germanic Linguistics Roundtable 2012 und des Sociolinguistics Symposium 19. Unterschiedliche Teilbereiche wurden 2011/12 auf eingeladenen Vorträgen an verschiedenen Universitäten (Stuttgart, Regensburg, HU Berlin, Berkeley, Yale, Tufts, Stockholm, Austin) vorgestellt. 